\title{
Thymoma Inducing Superior Vena Caval Syndrome
}

Najdat Bazarbashi*, Sohail Siddique, Abdelhameed Elsayed, Tamer Mohammed, Ahmad Alshammari and Christos Alexiou

Academic \& Training Affairs, Prince Sultan Cardiac Center, Price Sultan Military Medical City, Ministery of Defence, Riyadh, Kingdom of Saudi Arabia

\begin{abstract}
Thymomas are one of the most common tumors of the anterior mediastinum afflicting adult population. Approximately half of the patients have no complaints, and the diagnosis is, made incidentally on the chest radiograph done for different issues. However, symptoms may arise from compression of the mass on the trachea, the recurrent laryngeal nerve, the esophagus, the superior vena cava or other mediastnal structures. Invasive thymoma with transcaval extension to the right atrium is a rare cause of superior vena cava syndrome.
\end{abstract}

We present a case of a 63-year-old man presenting with dyspnea on exertion and facial and upper extremity swelling. Physical examination showed marked edema of face, upper torso and distention of superficial veins of the anterior chest wall and jugular veins. On elaborate investigations, it was revealed to be a case of Type B1 Masoka IVA invasive thymoma. The mass was removed employing open-heart surgery along with SVC reconstruction.

Keywords: Thymomas; Tumors; Pulmonology unit; Thoracotomy; Open-heart surgery; Pericardium

\section{Introduction}

Superior vena cava syndrome is an uncommon clinical entity that is usually a harbinger of a serious pathology. Thymoma producing SVC syndrome in an even rarer entity with sporadic reported cases. We present a case of a middle-aged man presenting with features of SVC syndrome and diagnosed with invasive thymoma as the causative lesion.

\section{Case Report}

The pulmonology unit referred a 63 years old man to us for features of SVC syndrome. He had noticed facial puffiness and upper extremity swelling about a year ago. This was gradual and painless in onset, but progressively increased and was most marked after lying down for long. Concomitantly he noticed exacerbation in already existing dyspnea being a known case of COPD and was NYHA-IV at presentation.

He underwent left thoracotomy and bullectomy/segmentectomy 15 years ago. He was a chain smoker and persisted with it even after hospitalization. He was on treatment for emphysema but with poor compliance. He was a well-built man with obvious swelling of upper extremities. His neck veins were engorged, and he was dyspnoeic with a wheezy chest. His systemic examination was unremarkable except a left posterolateral thoracotomy scar. His chest X-Ray showed a homogenous opacity in the upper anterior mediastinum and characteristics of emphysematous lung disease. Transthoracic echo showed a $2.5-1.0 \mathrm{~cm}$ mass in right atrium with central opacification. Transesophageal echo revealed a large heterogeneous mass in the SVC and extending into the right atrium. The mass was occupying most of the visible portion of the SVC with flow obstruction and dilatation of SVC. There were mobile filamentous components on the atrial part of the mass. (Impression: SVC thrombus extending into the right atrium with flow obstruction). Chest MRI showed a mass consistent with thymoma and invading the SVC, RA, and Innominate vein (Figures 1-3). The workup done for the possibility of subclinical Myasthenia Gravis turned out to be negative. Percutaneous transvenous tissue biopsy showed it to be type BI Masoka IVA invasive thymoma. After active consultations with interventional radiologist, oncologist, vascular and thoracic surgeons, we decided to remove the tumor employing open-heart surgery.

The patient was kept on therapeutic heparin while being prepared for surgery. Surgery was contemplated through a full median sternotomy. We found a very large darkish red mass occupying the entire anterior upper mediastinum and the upper half of pericardium (Figures 4 and 5). It was multilobulated and firm in consistency (Figure 6). We dissected the mass en bloc along with pericardium. The Innominate vein was clear proximally and was cannulated along with IVC and ascending aortic cannulation for Cardiopulmonary bypass after full heparization at this stage.

The remaining part of the procedure was carried out on the extracorporeal circuit with warm perfusate and beating heart. The SVC was incised longitudinally all along including Right Atrial appendage and the remaining tumor was removed from it, RA and Innominate vein (Figure 7). The SVC was reconstructed with an on lay pericardial

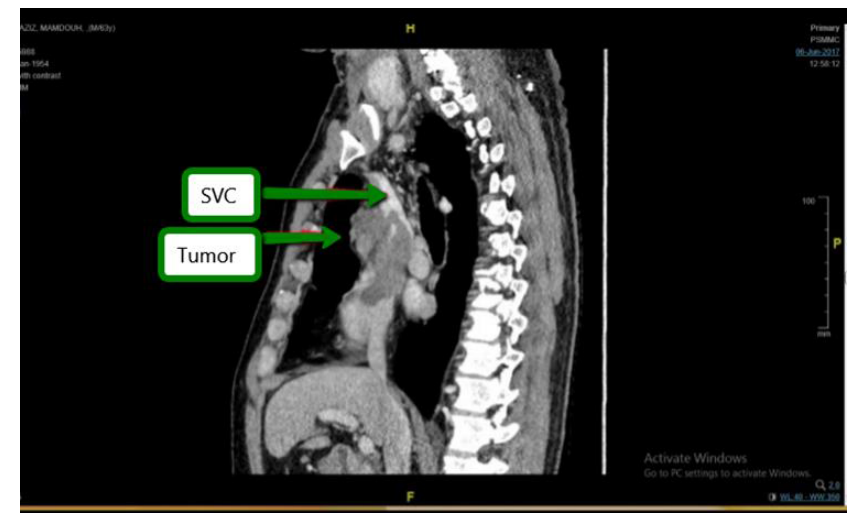

Figure 1: Mass invading SVC and right atrium.

*Corresponding author: Najdat Bazarbashi, Academic \& Training Affairs, Prince Sultan Cardiac Center, Price Sultan Military Medical City, Ministery of Defence, Riyadh, Kingdom of Saudi Arabia, Tel: 4783000; E-mail: N.bazarbashi@gmail.com

Received March 07, 2018; Accepted March 30, 2018; Published April 06, 2018

Citation: Bazarbashi N, Siddique S, Elsayed A, Mohammed T, Alshammari A, et al. (2018) Thymoma Inducing Superior Vena Caval Syndrome. J Vasc Med Surg 6 362. doi: 10.4172/2329-6925.1000362

Copyright: (c) 2018 Bazarbashi N, et al. This is an open-access article distributed under the terms of the Creative Commons Attribution License, which permits unrestricted use, distribution, and reproduction in any medium, provided the original author and source are credited. 
Citation: Bazarbashi N, Siddique S, Elsayed A, Mohammed T, Alshammari A, et al. (2018) Thymoma Inducing Superior Vena Caval Syndrome. J Vasc Med Surg 6: 362. doi: 10.4172/2329-6925.1000362

Page 2 of 3

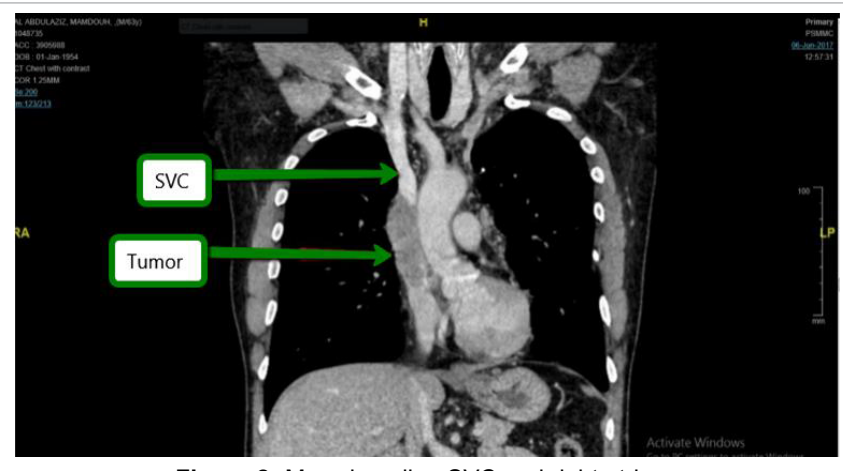

Figure 2: Mass invading SVC and right atrium.

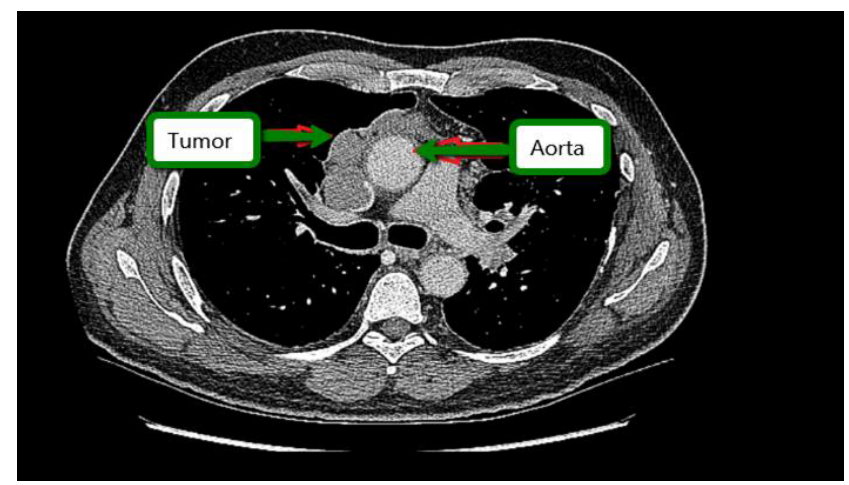

Figure 3: Mass encircling aorta

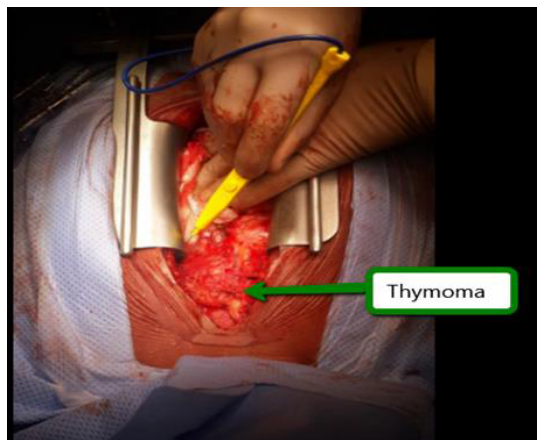

Figure 4: Mass occupying the entire anterior upper mediastinum.

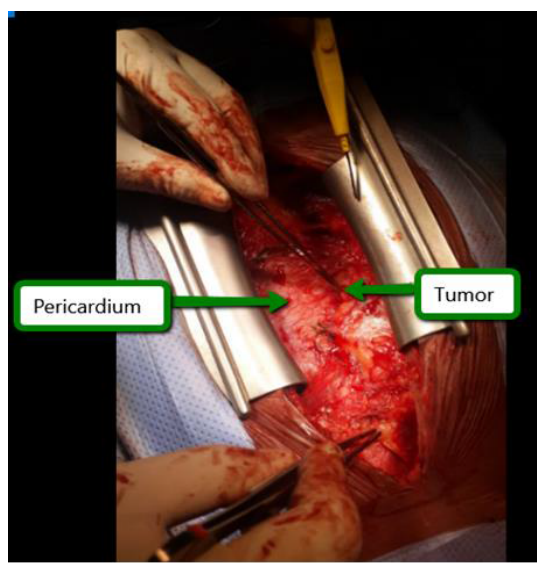

Figure 5: Mass occupying the upper half of pericardium patch (Figure 8). The patient was weaned of CPB uneventfully, and TEES showed patent SVC, Innominate vein, and RA. The patient had a smooth postoperative stay, and he was sent home on the $7^{\text {th }} \mathrm{POD}$. His upper extremity edema vanished altogether. However, SOB improved only to NYHA 1 as his COPD persisted. He was referred to oncology three months postoperatively, and appropriate therapy was instituted (Figure 9).

\section{Discussion}

Thymoma is an epithelial tumor of the thymus that accounts for nearly half of all primary tumors of the anterior mediastinum. Thymomas are perhaps the most prevalent of the mediastinal tumors accounting for about $45 \%$ of the mediastinal masses in adults [1,2].

Superior vena cava (SVC) syndrome may arise because of any pathology inducing obstruction of blood flow through the SVC. This can be an outcome of invasion or external compression of the SVC by a surrounding aetiology from the lymph nodes, lung, thymus, or

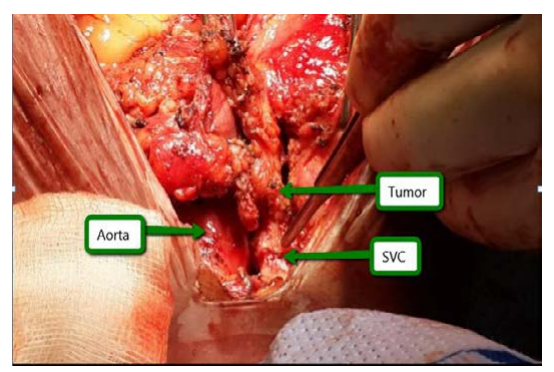

Figure 6: Multilobulated tumor

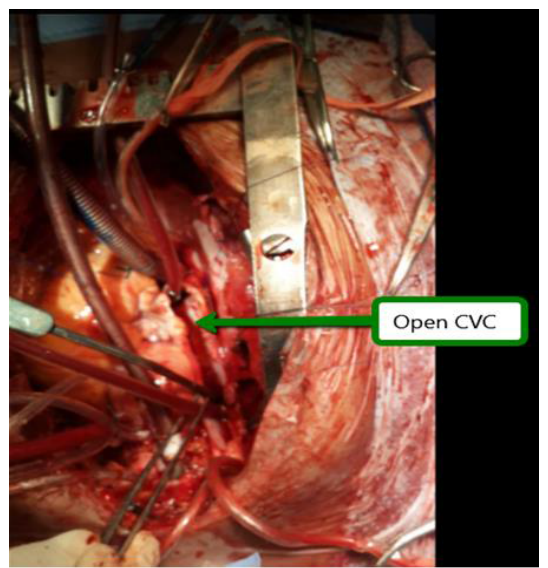

Figure 7: Tumor removal from SVC and Innominate vein.

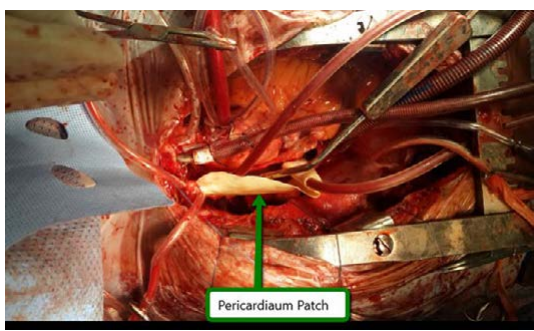

Figure 8: Pericardial patch SVC reconstruction. 


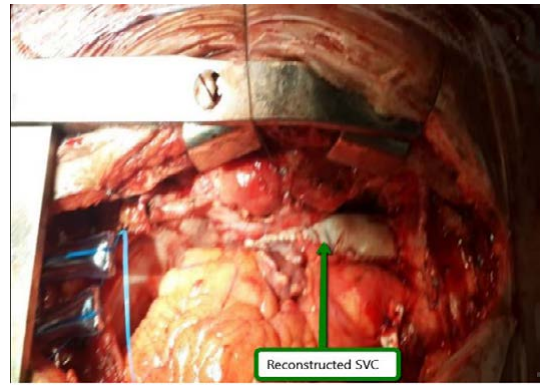

Figure 9: Reconstructed SVC.

other mediastinal tissues, or by thrombosis of blood within the SVC. Many times it is a combination of both external compression and thrombosis [3]. Most thymomas with cardiac involvement are limited to the pericardium, and very few cases of transcaval extension with intracardiac involvement have been reported.

Thymoma is a rare neoplasm primarily arising within the anterior mediastinum. Invasive thymoma commonly invades surrounding thoracic structures such as the heart, lungs, and vessels causing compressive symptoms [4]. SVC obstruction sets in usually because of external compression and uncommonly from transcaval thymoma encroachment [5].

About $60 \%$ to $90 \%$ of SVCS cases have been reported to be caused by the extrinsic compression or invasion of the SVC by malignant tumors, such as non-Hodgkin's lymphoma and small cell lung cancer. The de novo thrombosis developed by long-term indwelling intravascular devices and hypercoagulable states are considered as an essential etiology [6]. The main diagnostic modalities include CT scan and MRI, which may also delineate the mass at length, and the possibility of a successful resection. Tissue biopsy remains the gold standard not only for a definitive diagnosis and staging but also for the choice from various treatment modalities available. Tissue diagnosis and staging will also determine the prognostic age of the patient [7]. Surgery is aimed at restoration of blood flow in the superior vena cava to the RA and complete tumor excision as was done in our patient. The invading mass can be excised clear of SVC and RA without the need for any reconstruction. However, if needed, the continuity can be restored with an on lay pericardial patch (as was in our case), autologous vein, or PTFE grafts. Complete excision of the invading tumor is mandatory to be followed by appropriate radiotherapy or chemotherapy as indicated.

Post-operative detailed biopsy also reported it to be a case of type BI Masoka IVA invasive thymoma. These tumors are characterized by CD5 lymphocyte predominance, in-conspicuous epithelial cells positive for P63 expression and cytokeratin AE1/AE3 using the World Health Organization histologic classification [8]. The Masoka classification was stage IVA as there was pleural and pericardial invasion with no evidence of lymph nodes involvement or distant hematogenous spread [8]. Both histologic characteristics and tumor staging are independent prognostic factors. The invasiveness of a type B1 thymoma is $73.3 \%$, and the potential to invade greater vessels is $6.6 \%$ [8]. Our patient was undergoing sessions of radiotherapy as deemed suitable by the Oncologist.

\section{References}

1. Anupama K, Loehrer PJ (2004) Thymoma and thymic carcinoma: therapeutic approaches. Clin Lung Cancer 6: 28-32.

2. Kun-Eng L, Shen TC (2017) Surgical Treatment of an Invasive Thymoma with Intracaval and Intracardiac Extension. Acta Cardiol Sin 33: 204-206.

3. Garcia Monaco R, Bertoni H, Pallota G, Lastiri R, Varela M, et al. (2003) Use of self-expanding vascular endoprostheses in superior vena cava syndrome. Eur J Cardiothor Surg 24: 208-211.

4. Ashwad A, Wong I, Korniyenko A, Ivanov A, Worku B, et al. (2016) Superior vena cava syndrome from an invasive thymoma with transcaval invasion to the right atrium. J Surg Case Rep.

5. Memduh D, Sarvar S, Cekrezi B, Kaba E, Bakir B, et al. (2008) Cardiac metastasis from invasive thymoma via the superior vena cava: cardiac MRI findings. Cardiovasc Intervent Radiol 31: 209-212.

6. Kim HJ, Cho SY, Cho WH, Lee DH, Lim DH, et al. (2013) An unusual case of superior vena cava syndrome caused by the intravascular invasion of an invasive thymoma. Tuberc Respir Dis (Seoul) 75: 210-213.

7. Kim DJ, Yang WI, Choi SS, Kim KD, Chung KY (2005) Prognostic and clinica relevance of the World Health Organization schema for the classification of thymic epithelial tumors: A clinicopathologic study of 108 patients and literature review. Chest 127: 755-761.

8. Rena O, Papalia E, Maggi G, Oliaro A, Ruffini E, et al. (2005) World Health Organization histologic classification: an independent prognostic factor in resected thymomas. Lung Cancer 50: 59-66. 